\title{
APOYO AL DESARROLLO ECONÓMICO PRODUCTIVO Y SOPORTE EMPRESARIAL EN ZONAS MARGINALES DE LIMA: IMPLEMENTACIÓN DEL PROYECTO CAM1
}

\section{Introducción}

$\mathrm{H}$ emos considerado pertinente presentar la experiencia del proyecto Comunidad Autónoma de Madrid 1 (CAM1), primera iniciativa de desarrollo económico que la Universidad Católica Sedes Sapientiae (UCSS) realizó a través de su Centro de Asesoria Empresarial (CEASE), ya que muestra nítidamente el espiritu que está detrás de todas las obras que intentamos realizar, obras centradas en la persona humana, sus valores y sus posibilidades, asi como en lo real y tangible de nuestra visión y nuestros vínculos con la sociedad y su destino. Es esta experiencia, efectivamente, la primera acción; sin embargo, esta ha de marcar a las demás y, en general, a toda nuestra comunidad universitaria, mostrando a la colectividad que nuestros proyectos expresan en bechos concretos las propuestas conceptuales que compartimos en las aulas. 


\section{Presentación institucional}

La UCSS desde el comienzo de sus operaciones, abril del año 2000 , intenta formar hombres libres preparados para afrontar el reto de responder a problemas y exigencias de la realidad. Para lograr este objetivo, no se limitará a la formación académica, sino que promoverá el fortalecimiento del tejido social del cono norte de Lima. Así, en agosto del mismo año, abre el CEASE, que tendria que responder, desde sus inicios, a las demandas de un universo de microempresas y a los requerimientos de más de un millón de jóvenes que claman por comperitividad, empleo y valores.

\subsection{Recursos}

El principal recurso de una universidad es su comunidad de docentes y alumnos. En particular, la UCSS cuenta con dos Facultades, una de Ciencias Económicas y otra de Educación, ambas dotadas de equipos de docentes con experiencia académica y profesional. Los estudiantes son vecinos representativos del Cono Norte de Lima: provienen de hogares con limitaciones cconómicas y formativas; sin embargo, apuestan por su proyecto educativo en la UCSS. Ambos actores, docentes y alumnos, miran con responsabilidad los problemas que aquejan al resto de pobladores de la zona y, con facilidad, comprometen sus capacidades en los distintos proyectos que la universidad formula para atenuar y corregir las dificultades económicas, sociales y morales.

Destaca también el rol del Gran Canciller de la Universidad, el Obispo de la Diócesis de Carabayllo (el Obispo del Cono Norte de Líma), principal promotor de la UCSS y lider de una feligresía comprendida por 41 parroquias en una zona con más de dos millones de habitantes. El Cono 
Norte nace hace más de cuarenta años atrảs, y la iglesia fue determinante en su crecimiento y organización. Alrededor de sus parroquias se desarrollaron actividades económicas como pequeños talleres, centros de capacitación, cooperativas, centros de salud, centros educativos yotras actividades no lucrativas.

Constituye también un recurso el acceso a esta red de actividades económicas vinculadas a las parroquias, donde se ubican no solo laicos, sino, además, religiosas y religiosos con espiritu empresarial, quienes, apoyados por los proyectos de la universidad, serán los principales protagonistas del cambio económico y social en esta parte de Lima.

Otro recurso importante de la UCSS es su enfoque estratégico, que permite alinear tanto las decisiones y políticas de su alta dirección, asi como los programas y actividades de los responsables de proyectos con los objetivos de largo plazo expresados en términos de desarrollo y equidad.

\subsection{Propuesta}

Nuestra universidad pretende fortalocer el tejido económico y social en su ámbito de influencia Previamente, se identificaron algunos sectores «dinámicos», denominados así por su mayor nivel de capitalización, mejor dirección, capacidad de generar empleos y posibilidad de desarrollo de nuevos mercacios. Esstos son los sectores de metalmecánica, confecciones, carpinteria, calzado, planchado y pintum, y agroindustria. En ellos se busca fomentar la asociafividad, integrarlos a cadenas productivas y articularlos con las grandes y medianas empresas de manera que se propicie una transferencia tecnológica, mejora deprocesos y financiación.

Se trata de potenciar la oferta existente, hacerla innovadora y mejorar su gestión: así lograremos mayores beneficios para los microempresarios y empleo para los jóvenes. 
Se seleccionó al sector confecciones para la intervención por tratarse de un sector donde el Perú tiene potencial para transformar las ventajas comparativas que posee en fibras y mano de obra en ventajas competitivas; ya que este sector es altamente intensivo en mano de obra, principalmente femenina y juvenil.

El proyecto, mediante el apoyo al taller de confecciones Santa Fe (ubicado en la zona urbano-marginal de Collique, distrito de Comas), buscó desarrollar actividades formativas y una mejora productiva que posibiliten un incremento tanto en los niveles de producción como en el nivel de empleo.

\subsection{Algunas alianzas}

Poner en marcha un proyecto con alto componente técnico exigió el desarrollo de vinculos y relaciones con grandes y medianas empresas del sector, así como con especialistas y profesionales de nivel, entre los que podríamos citar a las empresas Textil San Cristóbal, Nettalco, Textil La Mar y a los ingenieros Ricardo Gómez y Fernando Rosales, dos de los mejores profesionales en operaciones de confecciones del país. Fue muy importante también la relación con el padre Claudio Chouniard, de los clérigos de San Viator, responsable del Taller Santa Fe, quien apostó por el proyecto y puso a disposición su infraestructura y organización. Finalmente, destacamos la función del Centro de Estudios y Solidaridad con América Latina (CESAL) y de la Comunidad Autónoma de Madrid (CAM) como facilitadores y proveedores que anspiciaron con generosidad y flexibilidad esta iniciativa. 


\subsection{Relevancia de la intervención}

Se han identificado más de mil unidades productivas de confecciones en el Cono Norte de Lima, cerca de un $90 \%$ de ellas son microempresas y adolecen de dificultades para alcanzar estándares de productividad y calidad, asi como de acceso a mercados sofisticados. Por ello, la iniciativa en el Taller Santa Fe pretende ser no solo una experiencia, sino una estrategia que posibilite definir una ruta de crecimiento y una ruptura con el estancamiento.

\section{Metodologia}

Por medio de los siguientes componentes: soporte empresarial y comercial, y desarrollo económico productivo, el proyecto encara tanto las necesidades de mejora en capacidades y procesos del taller, así como la preparación de jóvenes para su inserción laboral como maquinistas especializados en plantas industriales de confecciones de prendas de vestir.

\subsection{Desarrollo económico productivo}

Se transformó el pequeño Taller Santa Fe en un centro de formación y producción en confecciones para capacitar técnicamente a jóvenes que no tienen posibilidades de acceder a una formación profesional y, de esta forma, como objetivo final, insertarlos laboralmente en una pequeña, mediana o gran empresa o brindarles las habilidades para que creen su propia unidad productiva rentable.

Asimismo, este centro se constituirá en una entidad local que estimularí a los microempresarios a orientarse hacia el trabajo asociativo con las medianas y 
grandes empresas que operan en el sector. Estas últimas empresas demandan habitualmente personal técnico especializado en oficios especificos, y servicios de costura y acabados dentro de sus procesos productivos. Las microempresas, individualmente y con los estándares actuales, no pueden asumir pedidos de exportación directos por su falta de acceso a información, por su baja calidad y productividad, por sus pobres capacidades de financiación y comercialización.

\subsection{Soporte empresarial y comercial}

Nos propusimos estudiar con profundidad las necesidades técnicas de los pequeños productores del sector, así como las barreras estructurales para el crecimiento productivo y asociatividad entre ellos. Para ello, se realizó una investigación sobre las potencialidades del sector confecciones, sus posibilidades de articulación y desarrollo tanto de las relaciones comerciales como laborales entrelas pequeñas y grandes empresas. Esto se realizó mediante contactos directos y presentación del proyecto a empresas del sector, muchas de las cuales estuvieron interesadas en demandar mano de obra calificada y servicios de confecciones mediante subcontrata.

Con la finalidad de sensibilizat e informar a los microempresarios y emprendedores del sector se desarrollaron eventos y jornadas de capacitación. Finalmente, se desarrolló un softuare para el control de la producción y eficiencias en los pequeños talleres.

\subsubsection{La oportunidad}

El Perú posee una milenaria tradición textil que se remonta a épocas preincaicas. Las culturas Paracas y Chancay fueron dos exponentes de 
calidad en el tejido, manejo de la fibra y del teñido. En lo que se refiere a fibra de algodón, existen dos variedades importantes: la pima (de fibra extralarga), que crece en la zona norte del pais, y la Tangüis (de fibra larga), que crece, principalmente, en Ica y Lima. Las zonas andinas de Ayacucho, Huancavelica, Cusco y Puno albergan el $80 \%$ de la población mundial de alpacas y el $60 \%$ de vicuñas, ambas especies son poseedoras de fibras finas y cotizadas.

El mercado americano es el principal destino de nuestras exportaciones y, a diferencia de México y el Caribe, que desarrollan operaciones de maquilado, es decir, solo de costura, las plantas peruanas desarrollan toda la cadena, dado que las principales empresas peruanas están integradas verticalmente desde el proceso de la hilatura hasta la confección, como se aprecia en el gráficon. ${ }^{\circ} 1$ :

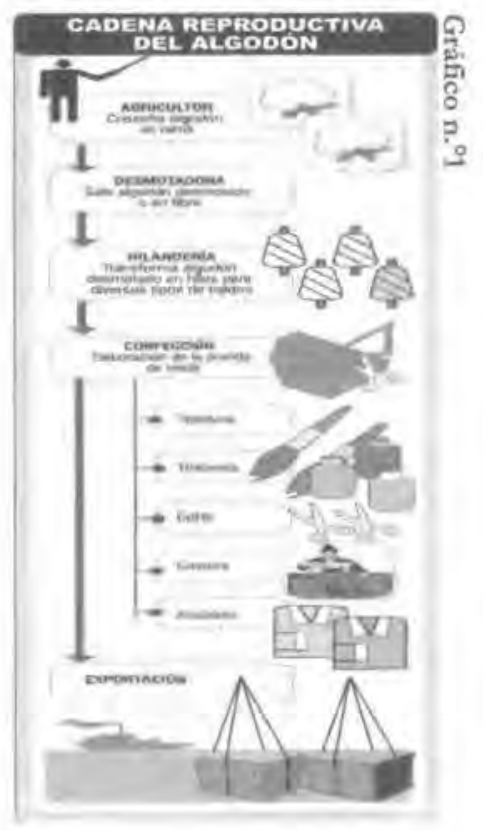


Asi, las exportaciones de prendas de vestir han pasado de ser del orden de los 80 millones de dólares a inicios de los noventa a más de 450 millones de dólares en el año 2000, y representan el $25 \%$ del total de las exportaciones no tradicionales y el $7.3 \%$ del total exportado por el pais en ese año. El destino principal de estas exportaciones son los Estados Unidos, hacia donde se envió casi el $80 \%$ de las exportaciones de confecciones peruanas en el año 2000, cuyo principal producto fueron los T-shirtsde algodón.

Por otro lado, la industria peruana de la confección se destaca por estar altamente integrada, dado que se produce en el país desde el algodón hasta las prendas terminadas, a diferencia de México y los países del Caribe, en donde la maquila se ha desarrollado fuertemente en los últimos años, lo que constituye competencia directz a las exportaciones peruanas en el mercado americano. En el caso peruano, tanto las grandes como las medianas empresas del pais destinan una parte importante de su producción al mercado externo; entre las más grandes, sobre todo, existe una plena integración vertical en el proceso de producción, que va desde la compra del hilo hasta el empaquetado del producto final.

\subsection{Equipamiento}

ElTaller Santa $\mathrm{Fe}$, hasta febrero de 2002, contaba con 32 máquinas y proyectaba tener 41 ; presentaba una capacidad de 4000 prendas como máximo y 800 prendas mensuales como mínimo. Actualmente, cuenta con 60 máquinas, de las que solo utilizan en promedio 24 , ya que todavía no han desarrollado una producción a plena capacidad; y están en la etapa de capacitación de costureras con la finalidad de poder operar a mayor capacidad en el corto plazo. La producción promedio actual es de 500 prendas diarias en T-shirt. 
sin embargo, su capacidad potencial debería ser como minimo el doble una vez que dispongan de las costureras capacitadas.

La UCSS cuenta desde su creación con instalaciones adecuadas para el desenvolvimiento apropiado de los cursos; aulas, carpetas, equipos roultimedia; asimismo, cuenta con una plana docente especializada en gestión empresarial e informática; sin embargo, una debilidad identificada es la limitación para disponer de docentes o especialistas en mejora de procesos. Esto ha sido superado gracias al apoyo brindado por las empresas medianas y grandes del sector, que han contribuido con los criterios técnicos necesarios para la orientación del proyecto.

\section{Bstrategia de intervención}

Las posibilidades de crecimiento y desarrollo del Perú pasan por la necesidad de promovet aquellos sectores productivos de base tecnológica que permitan que gran parte del valor añadido se quede dentro del país. Este es el caso del sector de confecciones que, adicionalmente, es intensivo en el uso de la mano de obra juvenil y femenina.

El Perú exporta 500 millones de dólares anuales en confecciones, el $70 \%$ dirigido a Estados Unidos, que es el principal mercado de prendas de vestir en el mundo (171 mil millones de dólares en el 2001).

Las oportunidades que brinda el Acuetdo de Preferencias Arancelarias Andinas (APTDEA) son tractivas en tanto facilitan un acceso con arancel cero al mercado norteamericano; sin embargo, se requieren medidas como inversiones en plantas de hilatura y teñido, mayor articulación entre las pequeñas y grandes empresas, capacitación de la mano de obra operaria y de directivos para el sector a fin de consolidarlo y expandirlo. Además, la vigencia de 
estas preferencias, solo hasta el 2006 y con carácter de no renovables, no incentiva las inversiones de largo plazo que serían necesarias para concretat el incremento de la capacidad de producción que permita mayores volúmenes exportables. Otra amenaza en el mediano plazo tiene que ver con el hecho de que en enero de 2005 vence el Acuerdo Multíibras, mediante el que se asignaban cuotas a paises con mucho potencial exportador en textiles, como China, estos podrian irnvadir con sus productos el atractivo mercado americano.

Ante estos desafíos, el Perú deberia definir un posicionamiento y explotar sus ventajas con respecto a sus principales competidores:

- Producción de productos de alto valor

- Integración de la cadena textil

- Provisión de servicio full package

- Calidad del algodón

- Cercanía del mercado EE.UU.

- Diversificar su oferta

- Explorar nuevos mercados, segmentos y clientes

- Prendas complejas, ventas por catálogo

- Responsabilizarse de la preproducción

Las perspectivas para el mediano plazo son de un crecimiento anual potencial de exportaciones de prendas superior al 20\%. Se deberá resolver los cuellos de botella, desarrollar nuevos mercados, diferenciarse y acceder a segmentos de mayor valor. 


\subsection{Asociatividad, clusters textiles y articulación productiva}

A la fecha, han sido, mayoritariamente, las grandes empresas las que han aprovechado estas oportunidades, pero sus posibilidades de maximizar sus ingresos se ven limitadas por el requerimiento de inversiones que no podrán asumir porque la mayorfa de ellas están sobreendeudadas. De este modo, solo articulándose estas con redes de pequeñas empresas competitivas podrian encarar, en mejores condiciones, las nuevas demandas de producción. Esto conviene a la gran empresa porque le permite concentrarse en procesos de mayor valor agregado, reduce sus costos fijos, gana capacidad y flexibilidad; y a la pequeña empresa porque recibe una transferencia tecnológica, accede indirectamente al mercado internacional, recibe financiación y mejora sus procesos; es decir, una solución gana-gana.

Entonces, formar redes de pequeñas empresas competitivas se convierte en parte de la solución al problema del desarrollo del sector de confecciones, esto no es tan fácil, ya que la mayoría de las PYME tienen limitaciones en sus procesos productivos y en su dotación de factores, especialmente de equipos y mano de obra especializada. Existe desconfianza y poca innovación. Surge, entonces, como una posibilidad, desarnollar políticas y acciones que incentiven la asociación de los pequeños productores en búsqueda del objetivo común de exportar y mejorar sus eficiencias. Este será un proceso que, acompañado adecuadamente por proyectos que prioricen la mejora productiva y la formación, logrará progresivamente incrementar la competitividad de las PYME y la calidad de sus relaciones. El objetivo final de política será desarrollar un clustertextil donde se encuentren los principales actores, como las grandes empresas, las pequeñas, los proveedores de insumos y servicios, la academia, las instituciones públicas, entre otros, y que, en forma 
conjunta, desarrollen relaciones comerciales con otras redes $y$ corporaciones globales. En orros términos, se trataria de identificar zonas geográficas determinadas con condiciones de albergar a todos estos actores y donde desarrollarían una dinámica económica y social de formación de una estructura productiva que posea una constante interrelación entre los actores que se aglomeran. Un requisito fundamental es que existan externalidades pecunarias e innovación constante.

\subsection{La estrategia en el Cono Norte}

En el Cono Norte de Lima, existen más de mil pequeñas empresas de confecciones, dentro de las cuales podemos identificaralgunas más capitalizadas y mejor gestionadas que las demás. Este es el caso del Taller Santa Fe, que tiene un mayor número de máquinas que una empresa pequeña en promedio y está mejor gestionada. La estrategia planteada propone identificar aquellas empresas "Campeonas" o edinamizadoras", fortalecerlas con una propuesta formativa productiva que permita no solamente dotar de equipos de mayor tecnología a la unidad productiva, sino de mano de obra calificada. De esta forma, transformamos una PYME ineficiente en una entidad competitiva; esta empresa generaría eslabonamientos con las más pequeñas y propiciaría relaciones virtuosas por medio del préstamo de equipos y entregando mano de obra especializada. El otro componente del proyecto, el soporte empresarial y comercial, pretende generar externalidades positivas a las pequeñas empresas de manera que valoren y se involucren en propuestas de asociatividad y mejora continuas.

Como muestra el gráfica $\mathrm{n} .{ }^{\circ} 2$, se pretende desarrollar relaciones entre las grandes empresas y las dinamizadoras. Estas han de materializarse 
por medio de la subcontrata y el suministro de mano de obra calificada para la costura. Un impacto indirecto de esta relación seria la nueva relación que puede desarrollar la empresa dinamizadora con el resto de las PYME más pequeñas facilitándoles equipos y mano de obra especializada. Fases siguientes de esta estrategia tendrian que ver con replicar la experiencia validada en este proyecto con otras empresas dinamizadoras y, en una etapa aun posterior, con intentar que la red fortalecida, y con mayor competitividad, intente desarrollar relaciones directas con empresas o redes internacionales.

ESTRATEGIA EN EL CONO NORTE

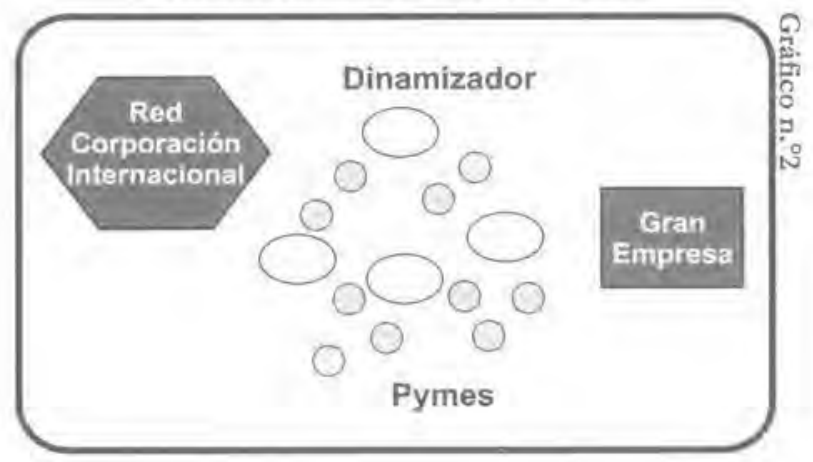

\section{Resultados cuantitativos del proyecto}

Los logros cuantitativos del proyecto se pueden expresar en el total de alumnos capacitados e insertados laboralmente, los contactos comerciales promovidos, las coordinaciones con grandes y medianas empresas, y otras actividades vinculadas. 


\subsection{Alumnos}

Delos 124 alumnos que egresaron, el $62.1 \%$ fue absorbido laboralmente por diferentes empresas, grandes y medianas, del sector; el $8 \%$ es microempresario y el $29.8 \%$ no está trabajando en lo que fue capacitado o no está trabajando por diferentes motivos.

CUADRO DE RESULTADOS DE LA CAPACITACIÓN

\begin{tabular}{|c|c|c|c|c|c|}
\hline $\begin{array}{c}\text { Periodos de } \\
\text { capacitación }\end{array}$ & $\begin{array}{c}\text { Alumnos } \\
\text { postulantes }\end{array}$ & $\begin{array}{c}\text { Alumnos } \\
\text { ingresantes }\end{array}$ & $\begin{array}{c}\text { Alumnos } \\
\text { graduados }\end{array}$ & $\begin{array}{c}\text { Alumnos } \\
\text { empleados }\end{array}$ & Mictoempreas \\
\hline $\begin{array}{c}\text { Primer curso: } \\
\text { Abr. -junio 2002 }\end{array}$ & 27 & 16 & 16 & 12 & 1 \\
\hline $\begin{array}{c}\text { Segundo curso: } \\
\text { Jul. - sep. 2002 }\end{array}$ & 45 & 20 & 20 & 15 & 2 \\
\hline $\begin{array}{c}\text { Tercer curso: } \\
\text { Oct. dic. 2002 }\end{array}$ & 70 & 22 & 17 & 15 & 2 \\
\hline $\begin{array}{c}\text { Cuarto curso: } \\
\text { Ene- marzo 2003 }\end{array}$ & 57 & 46 & 34 & 21 & 3 \\
\hline $\begin{array}{c}\text { Quinto curso: } \\
\text { Abr. - junio 2003 }\end{array}$ & 46 & 37 & 37 & 14 & 2 \\
\hline TOTAL & 245 & 141 & 124 & 77 & 10 \\
\hline
\end{tabular}

\begin{tabular}{|l|c|}
\hline Indice de empleabilidad (\%) & 62 \\
\hline Capacitación técnica (\%) & 87.9 \\
\hline
\end{tabular}

\begin{tabular}{|l|l|}
\hline Indice de microempresarios & 8 \\
\hline
\end{tabular}

\subsection{Contactos comerciales}

Microempresas que han logrado articularse con las grandes empresas:

1. Microempresa Raúl Vásquez Cevallos, ubicada en la segunda zona de Collique, Comas. Trabajó en el año 2002 brindando el servicio de confección de prendas para exportación a dos empresas grandes: 
- Allcotton, a cargo del ingeniero Marcelo Rodríguez (Gerente de Producción de la Empresa).

- Livees S. A. P., a cargo de la ingeniera Beatriz Chávez (Gerente de Producción de la Empresa).

2.Taller Santa Fe, ubicado en la tercera zona de Collique, Comas. Trabajó con las siguientes empresas:

- La Victoria S. A, a cargo de Mario Fioco (propietario).

- Allcotton, a cargo del ingeniero Marcelo Rodriguez (Gerente de Producción).

- Textil La Mar, a cargo del ingeniero Fernando Rosales (Gerente de Producción).

- Nexxus, a cargo del ingeniero Jorge Canales (dueño).

- Aaron, a cargo del ingeniero Carlos Vargas (Gerente de Producción).

- Copacabana, a cargo de Marlene Chauca (propietaria).

- Livees S. A. P., a cargo de la ingeniera Beatriz Chávez (Gerente de Producción).

3. Casa Betania, microempresa que fue contactada con el Taller Santa Fe para atender pedidos de Italia.

\subsection{Contacto con empresas para la inserción laboral de los alumnos egresados}

Progresivamente, de promoción en promoción, hemos ido desarrollando contactos con diferentes empresas del sector confecciones. Este es el caso 
de los contactos realizados con Textil San Cristóbal, Cotton Knitt, Topy Top, entre las principales.

\section{Inserción de los alumnos en pequeñas, medianas} y grandes empresas del sector confecciones

Las empresas que acogieron desde abril hasta diciembre a nuestros alumnos de los tres grupos son las sigaientes: Punto Export S.A., Orpi Confecciones, Extesa, Taller Santa Fe, Confecciones ECORPINDEL, La Victoria S.A., Confecciones Aaron S.A., Lima Garment, Incotex, Perú Fashion.

Asimismo, los alumnos de las últimas promociones (2003) fueron absorbidos por las siguientes empresas: Taller Santa Fe, Punto Export S.A., Orpi Confecciones, Nettalco, Tiara.

\section{CUADRO DE EMPRESAS CONTRATANTES}

\begin{tabular}{|c|c|c|c|c|}
\hline \multirow{2}{*}{ Curao } & \multirow{2}{*}{$\begin{array}{l}\text { Alurnnos } \\
\text { gradirados }\end{array}$} & \multicolumn{2}{|c|}{ Contacto con empresas } & \multirow{2}{*}{ Mieroetrprenatios } \\
\hline & & Bropresa & Alumnow & \\
\hline \multirow{5}{*}{ Abr. - han 2002} & \multirow{5}{*}{16} & Punto Ėxport & 2 & \multirow{5}{*}{1} \\
\hline & & Orpi Confecciones & 1 & \\
\hline & & Extesa & 1 & \\
\hline & & Tallor Santa Fe & 9 & \\
\hline & & Total & 13 & \\
\hline
\end{tabular}




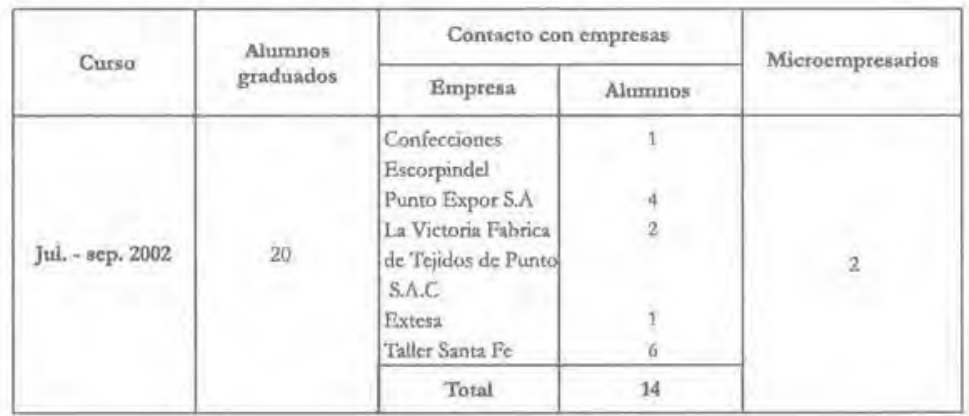

\begin{tabular}{|c|c|c|c|c|}
\hline \multirow{2}{*}{ Curso } & \multirow{2}{*}{$\begin{array}{l}\text { Alumnos } \\
\text { graduados }\end{array}$} & \multicolumn{2}{|c|}{ Contacto con empresas } & \multirow{2}{*}{ Microempresarios } \\
\hline & & Einpresa & Alumnos & \\
\hline \multirow{5}{*}{ Ene. - mat. 2003} & \multirow{5}{*}{34} & Taller Santa Fe & 8 & \multirow{5}{*}{3} \\
\hline & & Punto Export: & 2 & \\
\hline & & Orpi Confecciones & 8 & \\
\hline & & Nettalco & 3 & \\
\hline & & Total & 21 & \\
\hline
\end{tabular}

En total se han graduado 124 alumnos, 77 de ellos han sido absorbidos por empresas del sector, 10 tienen su propia empresa y 37 no han sido colocados, pues cumplen con el nivel necesario para ser colocados en una de las empresas del sector. 


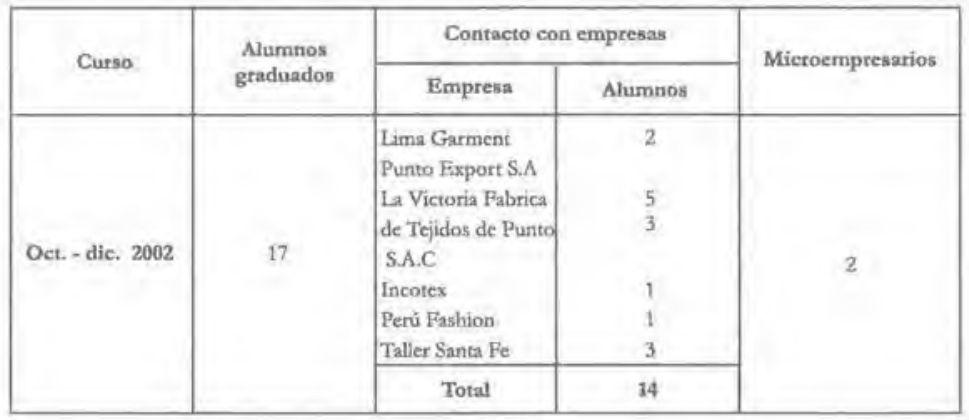

\begin{tabular}{|c|c|c|c|c|}
\hline \multirow{2}{*}{ Curso } & \multirow{2}{*}{$\begin{array}{l}\text { Alumanos } \\
\text { graduados }\end{array}$} & \multicolumn{2}{|c|}{ Contacto con empresas } & \multirow{2}{*}{ Microempreasrios } \\
\hline & & Empresa & Alumnos & \\
\hline \multirow{5}{*}{ Abs: - jun. 2003} & \multirow{5}{*}{38} & Punto Rsport & 3 & \multirow{5}{*}{2} \\
\hline & & Taller Santa $\mathrm{Fe}$ & 4 & \\
\hline & & Tiara & 2 & \\
\hline & & Otros & 5 & \\
\hline & & Total & 14 & \\
\hline
\end{tabular}

\section{Conclusiones y recomendaciones}

\subsection{Conclusiones}

- El sector confecciones posee un alto potencial para promover el desarrollo y crecimiento del país.

- La vigencia hasta el 2006 del APTDEA es una oportunidad para incrementar nuestra participación en el principal mercado de prendas de vestir, pero 
dada su condición de no renovable al 2006 y el término del Acuerdo Multifibras, que liberará de cuotas a China, ambos factores se presentan como serias amenazas que exigen decisiones y políticas para posicionar adecuadamente al Perú en el mercado textil utilizando sus ventajas difexenciales y capacidad para atender demandas sofisticadas.

- Una posibilidad para aprovechar mejor las oportunidades de APTDEA exige una articulación entre las pequeñas y grandes empresas; previamente, estas pequeñas unidades productivas deberian haber desarrollado un mínimo nivel de productividad y de gestión empresarial.

- El Cono Norte de Lima alberga a más de mil pequeñas empresas de confecciones. Existen empresas pequeñas más capitalizadas y mejor gestionadas que las demás. El proyecto de la UCSS propone promover el fortalecimiento de estas empresas denominadas «campeonas» o «dinamizadoras», las que serian apoyadas también para relacionarse con las grandes empresas, iniciar procesos de transferencia y, posteriormente, apoyar a las más pequeñas a través de préstamo de equipos de mayor tecnología y suministro de mano de obra especializada,

- El fortalecimiento del Taller Santa Fe corresponde al componente de desarrollo productivo-formativo; comprende la adquisición de equipos de alta tecnología para mejorar la capacidad productiva y elevar el nivel del entrenamiento de la mano de obra con métodos, con mejores prácticas de las grandes empresas y con instructores experimentados.

- El componente de soporte empresarial y comercial buscó profundizar el conocimiento de las PYME del sector en el Cono Norte destacando las barreras estructurales para el desarrollo de la asociatividad y crecimiento de sus empresas. Se organizaron seminatios y cursos orientados a sensibilizar e ilustrar a los microempresarios y emprendedores sobre la potencialidad de 
las articulaciones con las grandes empresas y los beneficios de una mayor competitividad.

- El desartollo de un software para el control de la producción tuvo como objetivo brindar un instrumento para desarrollar un eficiente monitoreo de las producciones de subcontrata y mejorar la planificación, respuesta rápida y aprovechamiento de la capacidad.

\subsection{Recomendaciones}

- Es importante destacar el papel de la dirección técnica, ya que en esta primera experiencia, para el logro de los objetivos, fue trascendente la propuesta formativa, el diseño y las recomendaciones de adquisición de equipos y materiales brindados por los consultores externos, importantes gerentes de operaciones de las principales empresas de confecciones del Perú. Sin embargo, esta consultoría no pudo acompañarnos con la frecuencia necesaria para asegurar mejores resultados en los indicadores de mejora productiva del taller. Consideramos que podríamos haber logrado acelerar el uso óptimo del total de la capacidad instalada del taller $y$, por consiguiente, un mayor empleo para jóvenes y mejores resultados económicos, si se hubiera utilizado con mayor intensidad la consultoria externa para reforzar la producción.

- El proyecto CAM1, como se aprecia en el gráfico n. 2 , ha intentado acercar a las grandes empresas con una unidad «dinamizadoran potenciándola y haciéndola más competitiva. Sería conveniente que posteriores iniciativas continúen desarrollando la estrategia que en el mediano plazo sentaria las bases para la configuración de un cluster textil.

- En esta línea, las investigaciones contempladas en el proyecto CAM2 intentan profundizar el conocimiento de las oportunidades internacionales 
mediante el estudio de las posibilidades de internacionalización del sector textil. En otro estudio sobre los conglomerados económicos, ha de evaluatse al sector de confecciones, entre otros, identificando la calidad de las relaciones entre sus pequeñas empresas, los niveles de innovación y factibilidad de desarrollo de asociatividad y clusters de firmas.

- El proyecto CAM3 (que ha de intervenir sobre otra pequeña unidad dinamizadora de confecciones, Presentación de Maria) debería alinearse en la formulación de su propuesta formativa con las acciones y políticas desarrolladas y validadas en el proyecto CAM1.

- Como se puede apreciar, se ha configurado una plaraforma estratégica que puede servir para soportar un sinnúmero de iniciativas complementarias que contribuyan en crear externalidades y que promuevan la asociatividad entre los actores de este sector tan dinámico y de mucho potencial. 